Brit. J. prev. soc. Med. (1974), 28, 63-64

\title{
A note on a 'close pairs' test for space clustering
}

\author{
P. G. SMITH AND M. C. PIKE \\ Department of Health and Social Security, Cancer Epidemiology and Clinical Trials Unit, 9 Keble Road. \\ Oxford, UK
}

Departments of Community Medicine and Paediatrics, University of Southern California School of Medicine, 2025 Zonal Avenue, Los Angeles, California 90033, USA

Lloyd and Roberts (1973) have recently proposed in this journal a test for spatial clustering. For the 57 cases of congenital limb defects diagnosed in singleton births in Cardiff over the three-year period 1964-67, they calculated the total number of pairs, $Z^{*}$, of cases whose places of residence at birth were less than a certain critical distance, $d$, apart. For comparison they took five control samples, each of size 57, drawn at random from the list of all singleton births in Cardiff during the same time period and, for each sample, they calculated the number of 'close' pairs. The average number of close pairs in the control samples was then used to test whether there was an excess of such pairs among children with limb defects, the significance level being derived by assuming that the number of close pairs in the control samples was drawn from a Poisson distribution.

We wish to point out here that the above test may be regarded as a special case of Knox's (1964) test, so that a randomization distribution of the number of close pairs may be defined and the expectation and variance under randomization of $Z^{*}$ may be easily derived. We also propose a method of analysis which is appropriate when a matched control sample (with matching, say, for social class) has been chosen.

\section{Equivalence to KNox's Test}

Suppose $n$ cases of the disease occur in the area during the period of study and $m$ controls have been selected (in the Cardiff study $n=57, m=5 \times 57=$ 285). The measure of space clustering is taken as the number of pairs of patients, $Z^{*}$, whose places of residence are less than some critical distance, $d$, apart.

If for each of the $(n+m) ! /(n ! m !)$ possible different selections of $n$ persons from the $(n+m)$ patients and controls we calculate the number of close pairs, $Z$, then this generated probability distribution of $Z$ may be regarded, by a combinatorial argument, as being the distribution under the null hypothesis from which $Z^{*}$ was drawn. The exact significance level can be evaluated by counting, or the expectation, $E(Z)$, and variance, $V(Z)$, of $Z$ may easily be computed to give an approximate test of significance.

This randomization test may be formulated in terms of the Knox test as follows. Create a 'pseudo onset time' for each person. Let each of the $n$ patients have onset on day 0 and the $m$ controls have onsets on days $1,2, \ldots, m$ respectively. The number of close pairs is then in the form of a Knox test if the critical space distance is defined as $d$ and the critical time distance as zero. The randomization of times of onset to places of onset is then exactly equivalent to randomly selecting $n$ persons from $(n+m)$ and counting the number of pairs close in space, and $E(Z)$ and $V(Z)$ are thus easily evaluated (Mantel, 1967).

This equivalence to the Knox test has been previously discussed by Mantel and Bailar (1970) and Mantel and Valand (1970).

\section{Matched CONTROLS}

Lloyd and Roberts (1973) noted that spurious spatial clustering may be detected if the disease being studied shows a relationship with, say, social class. In this case space clustering might merely indicate the tendency of persons in the same social class to live in the same area. Similarly, the age of patients in a study may be related to their area of residence. In these circumstances it would be desirable to choose a 'matched' control sample. Suppose the patients are divided into $k$ strata, $n_{i}$ patients falling into the $i$ th stratum $\left(\Sigma n_{i}=n\right)$, and the controls are similarly divided, $m_{i}$ persons falling into the $i$ th stratum $\left(\Sigma m_{i}=m\right)$.

The measure of space clustering is again given by the number of 'close' pairs, $Z^{*}$, among the $n$ patients, but the exact statistical permutational significance level of $Z^{*}$ is evaluated from the probability distribution generated by calculating 
the number of close pairs, $Z$, for each of the

$$
i \stackrel{k}{=}\left(n_{i}+m_{i}\right) ! /\left(n_{i} ! m_{i} !\right)
$$

possible different selections of $n$ persons from the $(n+m)$ patients and controls, such that $n_{i}$ persons are selected from the $\left(n_{i}+m_{i}\right)$ persons in the $i$ th stratum $(i=1,2, \ldots, k)$. An approximate test of significance may be obtained by computing $E(Z)$ and $V(Z)$ and assuming that

$$
\left(\left|Z^{*}-E(Z)\right|-0.5\right)^{2} / V(Z)
$$

is distributed as $\chi^{2}$ with 1 degree of freedom. The derivation of $E(Z)$ and $V(Z)$ is essentially straightforward, and detailed formulae are given by Pike and Smith (1973).

\section{An Allied Alternative Test}

An additional measure of spatial clustering for both of the above situations is given by the number of patients, $T^{*}$, involved in 'close' pairs. For example, three close pairs may involve six patients in three distinct pairs or only three patients all living close to each other. The expectation, $E(T)$, and the variance, $V(T)$, of the number of patients, $T$, involved in close pairs, using the above randomization approach, is also given by Pike and Smith (1973).
Fortran IV subroutines are available from the authors for the calculation of the expected values $\bar{D}$ and variances of the statistics suggested for the special case of $n_{i}=1$ and $m_{i}=m_{j}($ all $i, j)$. More $\overrightarrow{\bar{c}}$ general subroutines are being developed. A sub- $\overrightarrow{0}$ routine is also available which will generate the simulation distributions.

One of us (M.C.P.) is supported by Public Health Service Contract PH43-NCI-68-1030 within the Virus Cancer Program of the National Cancer Institute, $\stackrel{\infty}{\infty}$ National Institutes of Health, US Department of Health, Education and Welfare, Bethesda, Maryland 20014, USA.

\section{REFERENCES}

KNox, E. G. (1964). The detection of space-time interactions. Appl. Statist., 13, 25.

LloYd, S. and RoberTs, C. J. (1973). A test for space 9 clustering and its application to congenital limb $\overrightarrow{-}$ defects in Cardiff. Brit. J. prev. soc. Med., 27, 188.

MANTEL, N. (1967). The detection of disease clustering $ᄋ$ and a generalized regression approach. Cancer Res., 27, 209.

- and BaILAR, J. C. (1970). A class of permutational $\mathbb{D}$ and multinomial tests arising in epidemiological research. Biometrics, 26, 687.

— and Valand, R. S. (1970). A technique of nopparametric multivariate analysis. Biometrics, 26, 548.

Pike, M. C. and Smith, P. G. (1973). A case contral approach to examine diseases for evidence of contagiog including diseases with long latent periods. Biometric (In press). 